Política y Sociedad

ISSN-e: 1988-3129

\title{
Evidencia científica y alimentos funcionales: la regulación de las declaraciones de salud en la Unión Europea
}

\author{
Roberto López Mas ${ }^{1}$
}

Resumen. Este artículo plantea una caracterización del proceso regulador europeo de las declaraciones de salud, así como un análisis de la controversia generada en torno a sus requisitos metodológicos y evidenciales de fundamentación científica. A partir de la revisión de regulaciones, reglamentos y artículos científicos relevantes, se estudian las políticas epistémicas adoptadas por la Agencia Europea de Seguridad Alimentaria, su posible impacto en distintos ámbitos y las propuestas de estrategias alternativas presentadas por enfoques críticos. Desde la regulación, se ha definido una jerarquía evidencial y metodológica que otorga una importancia crucial al establecimiento de causalidad mediante ensayos controlados aleatorizados para la autorización de las declaraciones. Este estándar de prueba puede ser inadecuado para el estudio de los efectos de los ingredientes en las ciencias de la nutrición, el impulso a la investigación, el desarrollo y la innovación de los alimentos funcionales y la mejora de la salud individual y pública. Se concluye que la Agencia Europea de Seguridad Alimentaria puede necesitar reconsiderar las políticas epistémicas adoptadas hasta el momento, a través de la revisión de la priorización de sus objetivos reguladores y el análisis del conjunto de las consecuencias de cada nivel de prueba en los diferentes contextos.

Palabras clave: declaración de salud; regulación; fundamentación científica; ensayo controlado aleatorizado; causalidad; políticas epistémicas; nivel de prueba.

\section{[en] Scientific evidence and functional foods: health claims regulation in the European Union}

Abstract. This article proposes a characterization of the European regulatory process for health claims, as well as an analysis of the controversy surrounding the methodological and evidentiary requirements for scientific substantiation. The study of regulations and relevant scientific papers looks at three issues: the epistemic policies adopted by the European Food Safety Authority, their possible implications for different areas, and proposals for alternative strategies presented by critics' approaches. The hierarchy of evidence and methodologies defined by the regulation establishes that proving causality on the basis of randomized controlled trials is crucial for the authorisation of a claim. However, this standard of proof might be not suitable for investigating the effects of ingredients in nutrition science, promoting research, development and innovation in the functional foods sector, or improving individual and public health. The article concludes that the European Food Safety Authority may need to reconsider the epistemic policies hitherto adopted, by means of reviewing the prioritisation of its regulatory objectives and analysing the consequences for each level of proof in all contexts.

Keywords: health claim; regulation, scientific substantiation; randomized controlled trial; causality; epistemic policies; level of proof.

Sumario. 1. Introducción. 2. La Regulación de Nutrición y Declaraciones de Salud. 3. Controversia de la regulación europea. 4. Alternativas metodológicas y evidenciales. 5. Conclusión. 6. Bibliografía.

Como citar: López Mas, R.. (2021). Evidencia científica y alimentos funcionales: la regulación de las declaraciones de salud en la Unión Europea. Polít. Soc. (Madr.) 58(3), 74456. https://dx.doi.org/10.5209/poso.74456

Agradecimientos. La investigación presentada en este artículo ha sido posible gracias al apoyo financiero del Fondo Europeo de Desarrollo Regional de la Comisión Europea (FEDER) / Ministerio de Ciencia e Innovación de España - Agencia Estatal de Investigación (AEI) / Proyectos de Investigación "Estándares de prueba y elecciones metodológicas en la fundamentación científica de las declaraciones de salud" (FFI2017-83543-P) y "Las políticas epistémicas en la ciencia reguladora: evaluación de riesgos y evaluación de beneficios" (PID2020-113449GB-I00), así como de la ayuda predoctoral PRE2018-085271 y la Universidad de las Islas Baleares.

\section{Introducción}

En las sociedades contemporáneas, se ha producido en los últimos años un aumento del interés de los individuos por los alimentos con propiedades saludables. La función nutricional de los diferentes elementos de la 
dieta puede tener un papel importante para las elecciones de los consumidores. En este contexto, se ha generado un nuevo desarrollo dentro de la industria alimentaria: los alimentos funcionales. El concepto de alimento funcional surgió en Japón en la década de 1980, extendiéndose a otros países a finales de 1990 (Tarabella et al., 2019). En términos generales, los alimentos funcionales son productos que, además del valor nutricional que conllevan, aportan beneficios adicionales para conservar o mejorar la salud humana (Gregori y Gafare, 2012; Luján y Todt, 2018b; Tarabella et al., 2019; Todt y Luján, 2016). Los alimentos funcionales se pueden comercializar como tales mediante las declaraciones de salud.

Las declaraciones de salud son afirmaciones de los efectos beneficiosos para la salud —más allá de su aporte nutricional - que se espera que proporcione el consumo de un determinado ingrediente o alimento. También informan sobre su justificación científica y las posibles restricciones de uso, adoptando frecuentemente la forma de etiquetas en los envases de alimentos. Las declaraciones de salud pueden llegar a ser importantes para las elecciones que realizan los compradores, de modo que suelen estar sometidas a regulación en la mayoría de los países para poder ser comercializadas. En Europa, las exigencias reguladoras han cavado la gran mayoría de las solicitudes de declaraciones presentadas hasta la fecha, lo que ha generado un debate dentro y fuera de la comunidad científica con respecto a los requisitos de su autorización. El objetivo de este artículo es la caracterización del proceso regulador europeo de las declaraciones de salud y la controversia surgida en torno a los requisitos metodológicos y evidenciales de la sustanciación científica.

La investigación se ha llevado a cabo mediante el análisis cualitativo de regulaciones, reglamentos, guías y artículos científicos. En primer lugar, se han examinado los documentos referentes a fuentes primarias, publicados por la Comisión Europea (EC) y la Agencia Europea de Seguridad Alimentaria (European Food Safety Authority, EFSA). En este proceso, se han obtenido los datos relativos a los objetivos y los requisitos de la regulación europea, el método de evaluación de las solicitudes de declaraciones de salud, y las funciones y tareas de la agencia reguladora. Posteriormente, se han revisado los trabajos de fuentes secundarias, como los artículos de diferentes autores sobre las políticas epistémicas adoptadas por la EFSA. A través de estas investigaciones, se ha recopilado información de la jerarquía evidencial y metodológica de la regulación, así como del estándar de prueba constituido por el establecimiento de causalidad mediante ensayos controlados aleatorizados (randomized controlled trials, RCT). También se han examinado estudios críticos que, desde distintas disciplinas, han puesto en cuestión la adecuación del nivel de prueba definido por la EFSA. Tales trabajos han proporcionado datos sobre las consecuencias de los requisitos de la regulación en tres ámbitos: la investigación en las ciencias de la nutrición, la innovación de los alimentos funcionales y la salud individual y pública. Un análisis original ha identificado una interrelación entre cuestiones epistémicas y cuestiones pragmáticas del proceso regulador. Por último, se han estudiado las propuestas de los críticos sobre políticas epistémicas alternativas a las adoptadas hasta el momento.

Como conclusión se establece que las interrelaciones entre las cuestiones epistémicas y sus consecuencias pragmáticas justifican un enfoque consecuencialista con respecto a las elecciones metodológicas y los requisitos evidenciales de fundamentación científica. A partir de un análisis de los objetivos del proceso regulador de las declaraciones de salud, y sobre la base del conjunto de consecuencias de los distintos niveles de prueba, podría resultar conveniente que la EFSA considerara la modificación de su estándar de prueba.

\section{La Regulación de Nutrición y Declaraciones de Salud}

En Europa, la regulación de los alimentos funcionales fue aprobada en julio de 2007 con la implantación de la Regulación de Nutrición y Declaraciones de Salud (Nutrition and Health Claims Regulation, NHCR), un proceso promovido por la Comisión Europea (EC) y a cargo de la Agencia Europea de Seguridad Alimentaria (European Food Safety Authority, EFSA) (EFSA, 2011a; 2011b; 2016; 2017; European Parliament and Council, 2006). La NHCR afecta a todas las declaraciones de salud - tanto etiquetas de envases como publicidad o comunicaciones comerciales de todo tipo-, requiriendo una fundamentación científica para su aprobación. Un objetivo importante que tiene la EC con la implantación de la NHCR es proteger a los consumidores de la información falsa o confusa de las comunicaciones comerciales. Además, los efectos beneficiosos descritos en las declaraciones tienen que ser comprensibles para el consumidor promedio, que se considera razonablemente bien informado, observador y prudente. La NHCR también busca garantizar la unidad de mercado para los alimentos funcionales, fomentar la investigación y el desarrollo e impulsar la innovación industrial en este ámbito (Asp y Bryngelsson, 2008; Todt y Luján, 2016).

La NHCR exige que las declaraciones de salud tengan una fundamentación científica basada en toda la evidencia científica disponible - a favor o en contra, publicada o sin publicar- (EFSA, 2009; 2011a; 2011b; 2016; 2017; European Commission, 2008; European Parliament and Council, 2006), con la finalidad de justificar la aseveración de que el consumo de un alimento o ingrediente produce un determinado efecto beneficioso para la salud humana. No obstante, ciertas declaraciones de salud, más allá de su sustanciación científica, no pueden ser autorizadas desde la NHCR: a) las que informan sobre la prevención, tratamiento o cura de enfermedades; b) las presentadas en bebidas alcohólicas; c) las que ponen en cuestión el contenido nutricional 
de otros alimentos o la adecuación de una dieta equilibrada; d) las que sugieren que no consumir el producto podría afectar a la salud; e) las que se refieren a una tasa o cantidad específica de pérdida de peso; f) las que incluyen recomendaciones de profesionales de la salud concretos (Buttriss, 2019; EFSA, 2011b; 2016; 2017; European Commission, 2008; European Parliament and Council, 2002; 2006).

La operacionalización de todo el proceso regulador es llevada a cabo por la EFSA, que, en este contexto, tiene cuatro funciones principales: a) plantea a los solicitantes las recomendaciones sobre el proceso de autorización de las declaraciones de salud; b) define los criterios metodológicos que se tienen que adoptar para la fundamentación científica; c) evalúa la sustanciación de las declaraciones presentadas; d) mantiene un registro de las declaraciones examinadas (de Boer et al., 2014; Todt y Luján, 2016; 2017a; 2017b). La EFSA evalúa las declaraciones de salud a través de su comisión de nutrición, nuevos alimentos y alérgenos alimentarios (Panel on Nutrition, Novel Foods and Food Allergens, NDA Panel) (EFSA, 2011b; 2016), que valora toda la evidencia disponible para determinar hasta qué punto: a) el alimento o ingrediente está suficientemente caracterizado; b) el efecto nutricional o fisiológico queda suficientemente definido, siendo este relevante y beneficioso para la salud humana; c) se ha establecido una relación causa-efecto entre la ingesta del alimento y el efecto concreto. Cuando el resultado de la evaluación de cualquiera de estos tres puntos es desfavorable, la EFSA concluye que no se ha fundamentado científicamente la declaración de salud. Si, por otro lado, el resultado es positivo, procede a considerar en qué medida: d) la cantidad de consumo necesaria para obtener el efecto esperado es consistente con una dieta equilibrada; e) los grupos de estudio a partir de los que se ha obtenido la evidencia son representativos de los consumidores finales previstos; f) las condiciones o restricciones de uso son adecuadas (EFSA, 2009; 2011a; 2011b; 2017; European Commission, 2008). Los requisitos evidenciales definidos por la EFSA podrían ser diferentes en el caso de las declaraciones basadas en la esencialidad de los nutrientes (EFSA, 2016; 2017).

\subsection{Jerarquía evidencial y metodológica}

La EFSA ha establecido una jerarquización de la evidencia para operacionalizar la ponderación de toda la información científica disponible (EFSA, 2011b; 2017; European Commission, 2008). La jerarquía está organizada en función de los distintos tipos de datos que muestran en mayor o menor medida la existencia de un vínculo causal entre la ingesta del alimento y el efecto para la salud (Luján y Todt, 2018b). Si la evidencia presentada en las solicitudes no es suficiente para llegar a la conclusión de que se ha probado tal relación, las declaraciones de salud no pueden ser evaluadas positivamente por la EFSA y, por lo tanto, no se pueden autorizar (EFSA, 2009; 2011a; 2016; Martínez y Siani, 2017). De este modo, la regulación europea otorga una importancia crucial al establecimiento de causalidad, adoptando unos criterios para su consideración que coinciden en gran medida con los propuestos en su momento por sir Austin Bradford Hill (1965): solidez, consistencia, especificidad, plausibilidad biológica y relación dosis-respuesta.

La jerarquía de la evidencia se convierte en una jerarquía de las metodologías científicas sobre la base de que la evaluación de los distintos datos depende del nivel de confianza en la metodología específica utilizada en cada caso (EFSA, 2017; Luján y Todt, 2018b). En este sentido, la jerarquización de la EFSA parte de la idea de que cada tipo de estudio posee una capacidad inherente para la generación de datos relevantes con una solidez determinada (Todt y Luján, 2017a).

- Estudios de intervención en humanos

- Ensayos controlados aleatorizados

- Ensayos aleatorizados sin control

- Ensayos controlados sin aleatorización

- Otros estudios de intervención

- Estudios observacionales en humanos

- Estudios de cohorte

- Estudios de casos y controles

- Estudios transversales

- Otros estudios observacionales

- Trabajos de revisión de estudios en humanos

- Revisiones sistemáticas

- Análisis combinados

- Metaanálisis

- Otros trabajos de revisión

(EFSA, 2017)

La EFSA considera que las metodologías situadas en las posiciones más destacadas de la jerarquía proporcionan la evidencia más sólida para fundamentar las declaraciones de salud. En consecuencia, la mayoría de los datos relevantes para la regulación de las declaraciones proceden, en la práctica, de ensayos controlados 
aleatorizados (randomized controlled trials, RCT) (EFSA, 2010; Todt y Luján, 2017a; 2017b). Los RCT son pruebas en las que una muestra representativa de la población a ser estudiada se divide mediante un proceso aleatorio en dos grupos: el grupo de intervención, cuyos miembros reciben la sustancia bajo prueba, y el grupo de control, cuyos integrantes reciben un placebo (Aggett et al., 2005). Esta metodología surgió en el ámbito de la evaluación de fármacos para, posteriormente, ser transferida al campo de la nutrición (Blumberg et al., 2010). De hecho, los criterios de la EFSA para valorar la calidad de la estructura y el contenido de los RCT provienen de los definidos por el Consejo Internacional de Armonización de los requisitos técnicos para el registro de medicamentos de uso humano (ICH) (EFSA, 2017).

Los RCT se conciben como una metodología que permite tratar las asociaciones en nutrición como hipótesis científicas que se pueden someter a prueba por medio de experimentación, al generar resultados a partir de la diferencia que se produce entre los individuos que reciben la sustancia que está siendo estudiada y los que toman el placebo (Todt y Luján, 2017a; 2017b). Debido a su capacidad de establecer relaciones causaefecto sólidas entre la ingesta de ingredientes y beneficios para la salud, este tipo de estudio se considera el más idóneo en la mayoría de las regulaciones de las declaraciones de salud (Blumberg et al., 2010; EFSA, 2010; Heaney, 2008; Martínez y Siani, 2017; Mitchell et al., 2011; Richardson, 2012). En el marco regulador europeo, el establecimiento de causalidad mediante RCT constituye una condición básica para la autorización de las declaraciones, siendo un requisito de prueba fundamental formalizado a través de la jerarquía de las metodologías de la EFSA.

Los estudios observacionales pueden identificar una relación entre el consumo de un alimento y un efecto para la salud, pero no pueden distinguir si la diferencia observada en un grupo de individuos con unas pautas de consumo concretas se debe a un ingrediente específico o a otros factores que no se llegan a determinar ni medir (EFSA, 2010; Heaney, 2008; Martínez y Siani, 2017; Richardson, 2012). Por lo tanto, según la EFSA, los estudios observacionales no pueden probar causalidad sobre la base de una asociación estadística. Si bien en la regulación europea se puede proporcionar evidencia distinta a la generada por RCT para la sustanciación de las declaraciones de salud, esta no resulta decisiva en la evaluación. Los estudios en animales o los estudios in vitro se consideran complementarios, y solo se contemplan si hay RCT de los que se siga el establecimiento de una relación causa-efecto entre el ingrediente ingerido y el efecto esperado (EFSA, 2018; 2020).

\section{Controversia de la regulación europea}

Desde la implantación de la NHCR, la gran mayoría de las solicitudes han sido rechazadas (Richardson, 2012). La exigencia de datos procedentes de RCT tiene como consecuencia que solo una parte muy reducida de las solicitudes haya recibido una evaluación positiva de la EFSA (Todt y Luján, 2017b). En diciembre de 2012, 2.758 declaraciones de salud habían sido evaluadas, de las cuales solo 222 fueron autorizadas (Lenssen et al., 2018). En este contexto, se ha generado dentro y fuera de la comunidad científica una controversia respecto a la sustanciación de las declaraciones de salud. El problema se ha centrado en una cuestión: ¿qué significa fundamentación científica? Aunque se ha planteado una definición desde la regulación europea, esta es una pregunta abierta que admite más de una respuesta (Todt y Luján, 2016; 2021). Autores críticos con la práctica de la EFSA han defendido que su concepto de fundamentación podría no ser adecuado para la investigación en las ciencias de la nutrición. Además, tal elección reguladora podría tener consecuencias en el desarrollo, investigación e innovación de los alimentos funcionales, así como en el campo de la salud individual y pública.

\subsection{RCT en las ciencias de la nutrición}

Autores de las ciencias de la nutrición han argumentado que el nivel de prueba que se exige desde la regulación europea es ineficaz, tanto para la evaluación de las declaraciones de salud en los alimentos como para la investigación en su ámbito (Richardson, 2012; Verkerk, 2013). Aunque la EFSA (2009; 2011a) sostiene que se tienen en cuenta los procedimientos generales adoptados en los diferentes campos de investigación, sus críticos defienden que, en la práctica, no reconoce suficientemente el conjunto del conocimiento que se ha desarrollado en nutrición (Aggett, 2012; Todt y Luján, 2017b). Existe, por tanto, una divergencia entre la investigación en nutrición y la regulación europea. Mientras la EFSA otorga gran importancia al establecimiento de causalidad a través de RCT para la fundamentación científica, sus críticos ponen en cuestión la adecuación de tal requisito metodológico y evidencial para la evaluación de los efectos de la ingesta de nutrientes (Biesalski et al., 2011; Heaney, 2006; Visioli, 2012). En esta última línea, se ha defendido que los RCT podrían presentar complicaciones y limitaciones en relación con cuatro puntos principales: la identificación y el control de los factores de confusión, el diseño del grupo de control, la duración de los estudios y la medición de la eficacia de la sustancia.

Factores de confusión. Los críticos de la EFSA señalan que, en distintos RCT que someten a prueba el mismo ingrediente, los efectos fisiológicos producidos pueden no ser los mismos debido a las diferencias en las dietas de los participantes del estudio (de Boer et al., 2014; Todt y Luján, 2017b). La dieta se interpreta como 
un factor de confusión que puede influir en los resultados del ensayo, ya que se pueden generar interacciones entre el ingrediente bajo estudio y otros elementos alimentarios (Biesalski et al., 2011; Richardson, 2012). En consecuencia, para evaluar el efecto completo que puede tener el consumo de un ingrediente, resultaría necesario tener en cuenta todas las interacciones que se pueden dar con las distintas posibles dietas de los consumidores y otros factores de confusión difícilmente controlables, como las diferencias genéticas. A este respecto, se ha planteado que, aunque los RCT buscan neutralizar los factores de confusión, estos se podrían optimizar para obtener mayores beneficios fisiológicos a través de la combinación de distintos ingredientes (Aggett, 2012; Blumberg et al., 2010; Heaney, 2008; Verkerk, 2013). No obstante, desde la regulación europea, solo se contemplan los efectos individuales producidos por ingredientes individuales, de manera que no se toman en consideración los posibles beneficios generados por la interacción de varios ingredientes (EFSA, 2017). En última instancia, tanto las características propias de los alimentos como las exigencias metodológicas de la EFSA complican la identificación y el control de los factores de confusión en los RCT.

Grupo de control. La adecuación de los RCT en nutrición también se ha puesto en cuestión sobre la base de las limitaciones que presentan en relación al diseño de un control apropiado (Biesalski et al., 2011; Blumberg et al., 2010; Heaney, 2006; 2008; Visioli, 2012). Cuando se someten a prueba fármacos, los efectos de la sustancia se comparan con el estado de los miembros del grupo de control, en cuyo organismo no se halla ninguna presencia del fármaco. En los casos de los ingredientes, en cambio, no suele darse en los individuos una ausencia completa de los nutrientes. La comparación que se puede realizar entre el grupo de intervención y el de control en nutrición se basa en los diferentes efectos que tiene el ingrediente en niveles más altos o más bajos de presencia en el organismo (Richardson, 2012; Todt y Luján, 2017a). Además, los miembros del grupo de control deben recibir dosis del ingrediente que estén por encima del nivel de suficiencia —el límite bajo el cual se producirían efectos adversos por un consumo inadecuado del nutriente-, con el fin de no generar artificialmente deficiencias nutricionales en los participantes. Los individuos del grupo de intervención, por otro lado, suelen recibir cantidades muy superiores a las administradas al grupo de control. Sin embargo, la ingesta de nutrientes en dosis por encima del nivel de suficiencia puede no llegar a suponer una mejora medible en los efectos para la salud. Por lo tanto, según los críticos de la EFSA, el uso de los RCT en nutrición posee limitaciones: pueden someter a prueba la hipótesis de que la ingesta de ingredientes en cantidades por encima del nivel de suficiencia podría producir beneficios fisiológicos, pero no pueden estudiar los efectos adversos que se podrían generar por un consumo insuficiente de un determinado ingrediente porque no se puede diseñar un grupo de control adecuado.

Duración de los estudios. Los críticos también advierten posibles complicaciones en lo relativo a la duración que pueden llegar a tener los RCT en nutrición (Aggett, 2012; Biesalski et al., 2011; Blumberg et al., 2010). La ingesta de ingredientes no suele conducir a efectos inmediatos para la fisiología humana del mismo modo que ocurre con el consumo de fármacos (de Boer et al., 2014; Heaney, 2008; Visioli, 2012). Los efectos de los ingredientes pueden mostrarse solo tras una exposición constante durante años o décadas, o incluso pueden aparecer mucho tiempo después de que su ingesta haya cesado, tras largos periodos de latencia (Aggett et al., 2005). Los estudios sobre los efectos del consumo de determinados ingredientes, por consiguiente, tienen que ser de larga duración y gran complejidad. En este sentido, la propia EFSA reconoce que los RCT no son adecuados para estudios cuyos efectos vayan a ser observables solo a largo plazo.

Medición de la eficacia. Determinar la eficacia de un ingrediente puede ser difícil a causa de sus efectos múltiples, sutiles y duraderos (Heaney, 2008; Todt y Luján, 2017b). Aunque los RCT surgieron para analizar los efectos específicos, intensos y de corta duración de los fármacos, se hallan con mayores limitaciones ante la complejidad funcional de los ingredientes, según los críticos de la EFSA (Richardson, 2012). No se trata únicamente de que los efectos sutiles de los ingredientes sean difícilmente identificables mediante RCT, sino también de las complicaciones que presenta dicha metodología para estudiar la multifuncionalidad de los alimentos (de Boer et al., 2014). Además, existe una continuidad de reacciones diferentes para los distintos niveles de ingesta de un mismo ingrediente, por lo que, en muchos casos, no se conoce el consumo ideal para reducir o eliminar determinados factores de riesgo de desarrollar enfermedades (Aggett, 2012; Visioli, 2012). En definitiva, los RCT encuentran dificultades en la identificación y la medición de la eficacia de los ingredientes para producir beneficios para la salud.

La exigencia de datos de RCT podría ser inadecuada no solo para la investigación en nutrición, sino que también podría constituir un nivel de prueba ineficaz para la fundamentación científica de las declaraciones de salud por varias razones: a) las posibles interacciones entre los ingredientes y las dietas de los consumidores no se toman en consideración; b) la comparación entre el grupo de intervención y el de control puede ser limitada y compleja en su diseño; c) los periodos de latencia y los efectos a largo plazo del consumo de ingredientes requieren estudios de larga duración; d) determinados efectos múltiples o sutiles son difícilmente identificables; e) la ingesta ideal de ciertos ingredientes para que produzcan efectos que se dan en diferente medida dentro de una escala de consumo puede ser ignorada. En conclusión, los RCT podrían no ser adecuados para la generación de datos relevantes para el estudio de los alimentos funcionales, ya que presentan dificultades en la identificación y medición de la eficacia de los efectos de la ingesta de ingredientes. Incluso en los casos concretos en los que los RCT en nutrición son posibles, se podría requerir la coordinación de diversas pruebas, 
un gran número de participantes y mucho tiempo, entre otros recursos extraordinarios (Aggett, 2012; Biesalski et al., 2011; Gregori y Gafare, 2012; Richardson, 2012).

\subsection{Investigación, desarrollo e innovación de los alimentos funcionales}

Las comunicaciones comerciales sobre las propiedades saludables de los alimentos funcionales pueden constituir una oportunidad para las inversiones empresariales. Los productos con declaraciones de salud, al ofrecer a los consumidores un valor añadido, pueden otorgar a las empresas una ventaja estratégica respecto a sus competidores en el mercado (Bremmers et al., 2013; Bröring et al., 2017; Khedkar et al., 2016). En este sentido, la EC considera la NHCR como un estímulo para la investigación y el desarrollo de alimentos funcionales, así como para la innovación industrial (de Boer et al., 2014; European Parliament and Council, 2006; Khedkar et al., 2017). Ante un proceso regulador que evalúa la fundamentación científica de toda declaración de salud, los consumidores pueden tener más confianza en las afirmaciones sobre los beneficios fisiológicos de los alimentos (Aggett et al., 2005). En este contexto, podría haber un mayor interés en las inversiones en el sector de los alimentos funcionales, lo que podría llegar a promover la investigación y el desarrollo y, en última instancia, la innovación científica, tecnológica e industrial.

Sin embargo, según productores industriales, la NHCR puede haber generado el efecto contrario, teniendo un impacto negativo sobre la creatividad y la innovación en el ámbito de los alimentos funcionales (Biesalski et al., 2011; Bröring et al., 2017; de Boer et al., 2014; Lenssen et al., 2018; Richardson, 2012; Sadler, 2018; Verkerk, 2013). La improbabilidad de obtener una autorización para una declaración de salud podría explicar la reducción en el número de nuevas solicitudes que se ha producido en los últimos años (de Boer y Bast, 2015). Esto se debería a que los cálculos de los costes, beneficios y riesgos esperados en la inversión empresarial necesaria para someter productos nuevos a la NHCR pueden resultar desfavorables. Al final, se podría optar por no llevar a cabo la investigación requerida para desarrollar alimentos con beneficios fisiológicos. Desde la implantación de la regulación, el impulso a la investigación, el desarrollo y la innovación de los alimentos funcionales puede haber hallado complicaciones por tres factores principales: recursos limitados, beneficios reducidos e incertidumbre elevada.

Recursos limitados. La industria alimentaria en Europa se ha caracterizado tradicionalmente por una actividad investigadora y desarrolladora de baja intensidad, con una capacidad limitada para emprender procesos de producción y comercialización de alimentos funcionales nuevos (Bröring et al., 2017). Disponer de los recursos necesarios para la aprobación de las declaraciones de salud puede no haber sido posible para determinadas empresas. Como consecuencia, el gasto general realizado en la investigación y el desarrollo de alimentos funcionales puede haber sido reducido (Khedkar et al., 2017). Asimismo, productores industriales defienden que los estándares de la regulación europea son demasiado estrictos; exigen unos recursos financieros inasumibles para muchas empresas (Bremmers et al., 2013; de Boer y Bast, 2015).

Beneficios reducidos. Las empresas alimentarias con recursos limitados pueden presentar declaraciones de salud bajo el artículo 13.1 de la NHCR, según el cual una declaración puede estar fundamentada sobre evidencia científica aceptada de manera genera, al estar ampliamente documentada en literatura relevante y al haber consenso dentro de la comunidad científica (Bröring et al., 2017; European Parliament and Council, 2006; Richardson, 2012; Richardson y Eggersdorfer, 2015). De este modo, existe la posibilidad de desarrollar productos con declaraciones sin someterse a un proceso de evaluación excesivamente arriesgado o exigente en recursos (Khedkar et al., 2016; 2017). No obstante, el artículo 13.1 no concede ninguna exclusividad a los solicitantes, esto es, mientras una empresa asume todos los costes del proceso de autorización, sus competidores pueden posteriormente usar la declaración de salud sin gastos adicionales. La ausencia de un valor estratégico en las declaraciones bajo el artículo 13.1 podría conducir a una menor investigación por parte de las empresas alimentarias, debido a las expectativas de un beneficio económico limitado en un mercado donde se podría no disponer de ninguna ventaja respecto a los competidores (Bremmers et al., 2013; Mitchell et al., 2011). Al final, en lugar de una innovación radical, se podría dar el desarrollo de productos con reformulaciones simples y características similares.

Incertidumbre elevada. Se ha defendido que existe una falta de transparencia en diversos puntos de la regulación europea. Los críticos advierten la ausencia de una definición exacta de los requisitos de la autorización de las declaraciones, así como de la comprensión adecuada del consumidor promedio al que se hace referencia en la NHCR (Bröring et al., 2017). En esta línea, se ha destacado la opacidad de las opiniones científicas de la NDA Panel, que pueden no describir la evaluación completa de las declaraciones propuestas (Lenssen et al., 2018). En determinados casos de solicitudes rechazadas, la comisión no especifica por qué la declaración no está suficientemente fundamentada por los estudios presentados. Por lo tanto, en el futuro se podrían acabar realizando investigaciones con diseños similares a aquellos que ya han llevado a una evaluación negativa. La falta de transparencia del proceso regulador genera una incertidumbre que aumenta el riesgo de rechazo de las declaraciones y, por consiguiente, puede propiciar una disminución de las inversiones en las mismas.

En definitiva, la regulación puede haber limitado el impulso a la investigación, el desarrollo y la innovación de los alimentos funcionales. En primer lugar, un proceso estricto y exigente puede generar que las empresas 
alimentarias, tradicionalmente limitadas en recursos, realicen un gasto inferior en la creación y comercialización de productos nuevos. Además, las inversiones en las declaraciones de salud de menor coste y riesgo podrían no promover la innovación radical por las expectativas de unos beneficios económicos reducidos. Por último, la incertidumbre sobre el proceso de evaluación de las declaraciones puede aumentar el riesgo de las inversiones y, en consecuencia, generar una disminución en la investigación y el desarrollo. Es más, ante una práctica reguladora que ha rechazado la gran mayoría de las declaraciones propuestas, las empresas pueden reaccionar procediendo en torno a sus lagunas legales, a través de estrategias oportunistas (Bremmers et al., 2013; Bröring et al., 2017; de Boer y Bast, 2015). En tal situación, habría un impacto negativo aún mayor sobre la innovación de los alimentos funcionales, ya que se devaluarían los esfuerzos de los competidores que se someten a la NHCR.

\subsection{Consecuencias para la salud individual y pública}

La controversia sobre las exigencias metodológicas y evidenciales de la regulación europea adquiere especial relevancia en relación a las consecuencias finales para la salud pública. En los últimos años, ha aumentado el interés de los consumidores por los elementos de su dieta, de manera que sus elecciones alimentarias se pueden llegar a basar en su contribución al bienestar (Biesalski et al., 2011; Tarabella y Francescato, 2019; Tarabella et al., 2019; Verhagen et al., 2010). En este contexto, la NHCR podría promover un mayor conocimiento sobre nutrición y unos hábitos alimentarios saludables, al generar más seguridad en la validez de la información de las declaraciones de salud (Aggett et al., 2005; Richardson, 2012). Por tanto, la regulación puede tener consecuencias importantes para la salud individual y pública, dado que los consumidores pueden modificar sus patrones alimentarios incorporando productos comercializados como alimentos funcionales. (Todt y Luján, 2016; 2017b).

Los críticos de la EFSA sostienen que, aunque los requisitos de la fundamentación de las declaraciones protegen a los consumidores ante información falsa o confusa, también producen un incremento en los falsos negativos: se rechazan declaraciones de alimentos con beneficios para la salud (Luján y Todt, 2018a; 2018b; Todt y Luján, 2016; 2017a; 2017b). Esto sería consecuencia del estándar de prueba exigente constituido por el establecimiento de causalidad mediante RCT, pues determinadas declaraciones podrían no obtener la sustanciación científica necesaria para su autorización. En esta situación, decrecería la gama de productos con declaraciones permitidas, de modo que los consumidores dispondrían de menos información sobre los efectos de los alimentos funcionales y, por consiguiente, podrían no llegar a disfrutar de ciertos beneficios para la salud (Blumberg et al., 2010).

Autores del campo de la nutrición han defendido que el nivel de prueba de la regulación europea puede tener repercusiones negativas en las posibilidades prácticas de la investigación científica (Aggett, 2012; Blumberg et al., 2010; Mitchell et al., 2011). Considerando que la mayoría de la evidencia generada en nutrición se deriva de metodologías distintas a los RCT, su aplicación en cuestiones de salud pública puede resultar irrealizable sobre la base de los requisitos de la EFSA. Además, se podría acabar condicionando el conocimiento dentro de este ámbito, por lo que una parte significativa de los beneficios fisiológicos de los alimentos podría permanecer sin un estudio suficiente. Si bien la nueva ciencia podría dar lugar a un conocimiento que llevaría a una mejora de la salud de los consumidores, el impacto de la regulación sobre la investigación puede limitar tal resultado.

Asimismo, se ha señalado que la fundamentación de las declaraciones relativas a la reducción de riesgos para la salud puede ser excesivamente complicada en el marco regulador europeo (Todt y Luján, 2017a). La investigación de los efectos sutiles, múltiples u observables solo a largo plazo, así como la optimización de los factores de confusión en los estudios, pueden hallar limitaciones metodológicas. En consecuencia, los alimentos funcionalmente complejos pueden encontrar dificultades en su comercialización en Europa. Aunque las declaraciones basadas en las interacciones entre diferentes ingredientes podrían proporcionar mayores beneficios fisiológicos, los requisitos de la EFSA excluyen tal posibilidad. En última instancia, los consumidores podrían no llegar a beneficiarse de los alimentos funcionales que no se pueden investigar adecuadamente desde las exigencias metodológicas de la regulación.

Por último, la mejora de la salud individual y pública también podría verse limitada por la ausencia de impulso a la investigación, el desarrollo y la innovación de los alimentos funcionales (Khedkar et al., 2016; Richardson, 2012). Una falta de motivación de las empresas alimentarias para usar declaraciones de salud puede provocar una disminución de la inversión total en investigación, de tal forma que los beneficios fisiológicos que se obtendrían de un mayor estudio de los efectos de los ingredientes se verían limitados. En definitiva, tanto la posible inadecuación de las exigencias de la EFSA en la investigación en nutrición como el impacto negativo que puede haber tenido la regulación sobre el desarrollo de alimentos funcionales nuevos pueden, a largo plazo, acabar generando consecuencias importantes para la salud de los consumidores.

\section{Alternativas metodológicas y evidenciales}

A partir del análisis de la controversia, se puede identificar que las consecuencias del proceso regulador se han desarrollado en cuestiones epistémicas y cuestiones pragmáticas interrelacionadas. Más allá del impacto 
sobre las elecciones metodológicas en la investigación de los efectos de los ingredientes, se advierten posibles consecuencias económicas, sociales y de salud. Un estándar de prueba exigente para la sustanciación de las declaraciones podría acabar frenando la creación y comercialización de alimentos funcionales nuevos, así como la mejora de la salud pública. Al mismo tiempo, un menor gasto total de la industria alimentaria en productos con declaraciones podría conducir a una disminución de la investigación en nutrición. Por lo tanto, las políticas epistémicas adoptadas en la regulación pueden tener un impacto negativo sobre la inversión empresarial realizada en el ámbito de los alimentos funcionales, lo que, a su vez, puede acabar limitando el desarrollo de estudios en las ciencias de la nutrición.

El estado actual de la controversia se puede entender desde cuatro puntos generales que exponen algunos de los aspectos de la regulación más debatidos por autores de diferentes disciplinas:

- El rechazo de la gran mayoría de declaraciones de salud ha generado un interés por los requisitos $-\mathrm{y}$ la justificación de los mismos- de su autorización.

- La adecuación de las exigencias metodológicas y evidenciales de la fundamentación científica de las declaraciones se ha puesto en cuestión en diversos ámbitos.

- El nivel de prueba se ha considerado, según los críticos, ineficaz con respecto a objetivos epistémicos y objetivos pragmáticos del proceso regulador.

- Se ha planteado la necesidad de analizar políticas epistémicas distintas a las adoptadas hasta el momento por la EFSA.

En relación al último punto, se ha defendido que, en circunstancias complejas, los datos procedentes de RCT para el establecimiento de una relación causa-efecto sólida entre el consumo de un ingrediente y un determinado efecto para la salud pueden no estar disponibles (Aggett, 2012; Biesalski et al., 2011; Mitchell et al., 2011; Richardson, 2012). Cuando se requiere la caracterización completa de la dieta, la actividad física y otros factores del estilo de vida de los individuos desarrollados a largo plazo, es necesario contemplar políticas epistémicas diferentes a las exigidas por la EFSA. En esta línea, los críticos han presentado cinco propuestas principales basadas en valores epistémicos y valores pragmáticos para considerar alternativas metodológicas y evidenciales:

Totalidad de la evidencia cientifica. En los casos de ausencia de evidencia concluyente de RCT, se puede recurrir a la ponderación de la totalidad de los datos científicos disponibles para fundamentar las declaraciones (Biesalski et al., 2011; Mitchell et al., 2011). La idea de una jerarquía evidencial y metodológica es rechazada por los críticos, que plantean un sistema en el que toda la evidencia científica de alta calidad sea valorada como complementaria y con la misma relevancia para el establecimiento de causalidad (Aggett, 2012). Dado que cada metodología tiene tanto limitaciones como una determinada efectividad en la generación de datos, adquiere importancia la revisión crítica de la totalidad de la evidencia disponible, independientemente de su procedencia (Richardson, 2012). En términos generales, se sostiene que la relación entre la ingesta de un alimento y sus beneficios fisiológicos se puede probar mediante estudios con diseños diferentes.

Estudios observacionales. Críticos de la EFSA defienden que se puede inferir causalidad a partir de estudios observacionales, de acuerdo con los criterios de Bradford Hill para evaluar la solidez, plausibilidad y consistencia de las asociaciones (Aggett, 2012; Biesalski et al., 2011; Blumberg et al., 2010; Richardson, 2012). De hecho, cuando los RCT hallan límites éticos o la necesidad de recursos excesivos, los estudios observacionales constituyen la principal fuente de evidencia en la investigación en nutrición. El estudio de cohorte prospectivo a largo plazo se ha presentado como una alternativa razonable a los estudios de intervención. Si bien sus resultados pueden verse comprometidos por elementos extrínsecos no aleatorizados, se puede minimizar el riesgo de sesgos a través de la identificación y el control de todos los factores de confusión (Heaney, 2006; Visioli, 2012). De este modo, los estudios de cohorte prospectivos bien diseñados podrían generar evidencia sólida para la toma de decisiones reguladoras.

Evidencia mecanística. Otra propuesta destacable de los críticos es la atribución de una mayor relevancia a la evidencia mecanística para la fundamentación de las declaraciones (Aggett, 2012; Biesalski et al., 2011). Se arguye que el razonamiento mecanístico basado en evidencia puede llegar a establecer una relación causa-efecto entre la ingesta de un ingrediente y un beneficio para la salud. Aunque provenga de estudios en animales, estudios in vitro, u otros estudios en laboratorios, la evidencia mecanística de calidad debería ser considerada y analizada, pues, en su conjunto, podría caracterizar relaciones causales.

Estándares de prueba. Frente a un estándar evidencial exigente que procura proteger a los consumidores ante información falsa y preservar la credibilidad del proceso regulador, los críticos plantean la posibilidad de un estándar de prueba más permisivo que favorezca otros objetivos (Biesalski et al., 2011; Blumberg et al., 2010; Luján y Todt, 2018a; 2018b; Mitchell et al., 2011; Todt y Luján, 2016; 2017a; 2017b). Políticas epistémicas alternativas a las de la EFSA podrían priorizar el fomento de la innovación industrial, el impulso a la investigación multidisciplinaria, el estímulo al desarrollo de alimentos funcionales o la mejora de la salud individual y pública. Esta estrategia conllevaría la aceptación de un mayor nivel de incertidumbre en los datos relevantes para la toma de decisiones reguladoras, incrementando así los falsos positivos: se aprobarían más 
declaraciones para productos que no generan los efectos fisiológicos que afirman ofrecer. No obstante, también habría un descenso de los falsos negativos, por lo que se rechazarían menos declaraciones de alimentos funcionales. Por lo tanto, aunque los consumidores se encontrarían más desprotegidos ante las declaraciones falsas, según los críticos, se podrían llegar a alcanzar otros fines sociales, económicos o de salud.

Consecuencialismo. Se defiende que las políticas epistémicas definidas en la regulación deben ser el resultado de análisis explícitos que consideren sus consecuencias pragmáticas (Biesalski et al., 2011; Blumberg et al., 2010; Luján y Todt, 2018a; 2018b; 2021; Todt y Luján, 2017a). En este sentido, no solo es necesario estudiar las repercusiones de un estándar de prueba exigente para la sustanciación científica de las declaraciones, sino también el impacto que podría tener en los distintos ámbitos la aceptación de un nivel de evidencia más bajo. Por un lado, un nivel de prueba alto puede acabar limitando la investigación en nutrición, el desarrollo de alimentos funcionales nuevos y la mejora de la salud de los consumidores. Por otro, la adopción de un estándar evidencial menos estricto puede llegar a generar consecuencias negativas como la deslegitimación de las agencias y los procesos reguladores, la validación de la mala ciencia o ciertos efectos socioeconómicos. Por consiguiente, no existe una solución general en lo relativo a la selección de las políticas epistémicas, ya que el nivel de prueba apropiado depende de qué se esté regulando y en qué contexto. En definitiva, para determinar las políticas epistémicas adecuadas en cada caso, se deben considerar las diferentes consecuencias positivas y negativas que puede conllevar la autorización o el rechazo de declaraciones de salud concretas.

En resumen, frente a la exigencia de datos de RCT para el establecimiento de causalidad, los críticos de la práctica reguladora europea proponen alternativas metodológicas y evidenciales mediante: a) la revisión de la totalidad de la evidencia científica disponible; b) el reconocimiento de la importancia de los estudios observacionales; c) la atribución de una mayor relevancia a la evidencia mecanística; d) la consideración de un estándar de prueba menos estricto; e) el análisis de las consecuencias pragmáticas de las políticas evidenciales. Los primeros tres puntos se basan en criterios principalmente epistémicos que examinan las ventajas y los inconvenientes de los distintos tipos de estudios utilizados en nutrición y la ciencia reguladora. En cambio, las últimas dos propuestas tienen en cuenta, además, valores pragmáticos como los objetivos del proceso regulador o sus consecuencias últimas sobre la sociedad, la economía o la salud de los consumidores. En cualquier caso, los críticos sostienen que resulta necesario valorar estrategias diferentes a la adoptada por la EFSA para la sustanciación de las declaraciones.

\section{Conclusión}

Desde la implantación de la NHCR, la EFSA ha establecido una jerarquización evidencial y metodológica que exige la prueba de causalidad mediante RCT para la autorización de las declaraciones de salud. En este marco regulador, la gran mayoría de las solicitudes presentadas hasta la fecha han recibido una evaluación negativa, lo que ha contribuido a la generación de una controversia respecto a la fundamentación científica de las declaraciones. Autores de diversas disciplinas han destacado tres consecuencias principales de la estrategia reguladora europea: a) el requisito de datos de RCT puede ser inadecuado tanto en la investigación en nutrición como en la evaluación científica de las declaraciones; b) la NHCR puede haber tenido un impacto negativo sobre la investigación, el desarrollo y la innovación de los alimentos funcionales; y c) el nivel de prueba de la regulación podría llegar a limitar la mejora de la salud individual y pública.

Los críticos sostienen que, si bien los RCT pueden proporcionar los datos más sólidos para el establecimiento de relaciones causales, estos estudios no siempre son posibles o convenientes en la ciencia reguladora. Por lo tanto, es necesario valorar alternativas metodológicas y evidenciales que consideren la efectividad y las limitaciones de los diferentes tipos de estudios disponibles. Un estándar de prueba que acepte datos con un mayor grado de incertidumbre podría conducir a beneficios sociales, económicos o de salud. No obstante, las elecciones de las estrategias reguladoras deben ser, en todo caso, el resultado de análisis explícitos del conjunto de las consecuencias epistémicas y consecuencias pragmáticas de cada nivel de prueba en los distintos contextos.

Las interrelaciones entre las cuestiones epistémicas y sus consecuencias pragmáticas justifican un enfoque consecuencialista en ciencia reguladora: las elecciones metodológicas y los requisitos evidenciales para la evaluación de hipótesis deben fundamentarse sobre datos empíricos del impacto de cada nivel de prueba en los diferentes ámbitos. El conocimiento tiene que ser relevante para la toma de decisiones reguladoras. Si la evidencia disponible más concluyente no optimiza la obtención de beneficios - y la prevención de riesgos-, se debería estudiar la posibilidad de aceptar datos menos precisos o fiables. Tanto los objetivos reguladores como el conjunto de las consecuencias últimas deben determinar las políticas epistémicas adoptadas en cada contexto.

La EFSA puede hallarse en una situación en la que podría necesitar reconsiderar, en primer lugar, la priorización de los objetivos de la regulación europea. La protección de los consumidores ante información falsa y la preservación de la credibilidad del proceso regulador son fines que se han podido perseguir en detrimento de otros, como el fomento de la investigación científica, el impulso a la industria alimentaria o la mejora de la salud pública. Un replanteamiento de los propósitos de la NHCR requeriría cambios en las políticas epistémicas adoptadas hasta el momento. A partir de un análisis de los diferentes niveles de prueba, se podría concluir 
que unas políticas epistémicas distintas son más eficaces para: a) garantizar el cumplimiento de los objetivos reguladores priorizados; y b) minimizar las consecuencias no deseadas en diversos ámbitos. En tal situación, la EFSA debería considerar la modificación del estándar de prueba de la regulación, sobre la base de valores epistémicos y valores pragmáticos. La exigencia de datos causales de RCT tendría que ser revisada, contemplando la posibilidad de otorgar una mayor relevancia a la evidencia procedente de estudios observacionales o mecanísticos para la sustanciación científica de las declaraciones.

\section{Bibliografía}

Aggett, P. J. (2012): "Dose-response relationships in multifunctional food design: assembling the evidence", International Journal of Food Sciences and Nutrition, 63(S1), pp. 37-42. Doi:10.3109/09637486.2011.636344

Aggett, P. J., J. M. Antoine, N. G. Asp, F. Bellisle, L. Contor, J. H. Cummings, J. Howlett, D. J. G. Müller, C. Persin, L. T. J. Pijls, G. Rechkemmer, S. Tuijtelaars y H. Verhagen (2005): "Passclaim: consensus on criteria", European Journal of Nutrition, 44(Suppl. 1), pp. I/5-I/30. doi:10.1007/s00394-005-1104-3

Asp, N. G. y S. Bryngelsson (2008): "Health claims in Europe: new legislation and Passclaim for substantiation", The Journal of Nutrition, 138(6), pp. 1210S-1215S. Doi:10.1093/jn/138.6.1210S

Biesalski, H. K., P. J. Aggett, R. Anton, P. S. Bernstein, J. Blumberg, R. P. Heaney, J. Henry, J. M. Nolan, D. P. Richardson, B. van Ommen, R. F. Witkamp, G. T. Rijkers y I. Zöllner (2011): "26th Hohenheim Consensus Conference, September 11, 2010 scientific substantiation of health claims: evidence-based nutrition”, Nutrition, 27(10 Suppl.), pp. S1-S20. Doi:10.1016/j. nut.2011.04.002

Blumberg, J., R. P. Heaney, M. Huncharek, T. Scholl, M. Stampfer, R. Vieth, C. M. Weaver y S. H. Zeisel (2010): "Evidencebased criteria in the nutritional context", Nutrition Reviews, 68(8), pp. 478-484. Doi:10.1111/j.1753-4887.2010.00307.x

Bremmers, H. J., B. M. J. van der Meulen y K. Purnhagen (2013): "Multi-stakeholder responses to the European Union health claims requirements", Journal on Chain and Network Science, 13(2), pp. 161-172. Doi:10.3920/JCNS2013.1006

Bröring, S., S. Khedkar y S. Ciliberti (2017): "Reviewing the Nutrition and Health Claims Regulation (EC) No. 1924/2006: what do we know about its challenges and potential impact on innovation?", International Journal of Food Sciences and Nutrition, 68(1), pp. 1-9. Doi:10.1080/09637486.2016.1212816

Buttriss, J. (2019): "Health claims regulation: opportunities and challenges in Europe", en S. Astley, ed., Health claims and food labelling, Reino Unido, Royal Society of Chemistry, pp. 63-78.

De Boer, A. y A. Bast (2015): "Stakeholders' perception of the nutrition and health claim regulation", International Journal of Food Sciences and Nutrition, 66(3), pp. 321-328. Doi:10.3109/09637486.2014.986071

De Boer, A., E. Vos y A. Bast (2014): "Implementation of the nutrition and health claim regulation - the case of antioxidants", Regulatory Toxicology and Pharmacology, 68(3), pp. 475-487. Doi:10.1016/j.yrtph.2014.01.014

EFSA (2009): "Technical report: briefing document for Member States and European Commission on the evaluation of Article 13.1 health claims", EFSA Journal, 7(11):1386, pp. 1-10. Doi:10.2903/j.efsa.2009.1386

EFSA (2010): "Guidance on human health risk-benefit assessment of food", EFSA Journal, 8(7):1673, pp. 1-41. Doi:10.2093/j. efsa.2010.1673

EFSA (2011a): "General guidance for stakeholders on the evaluation of Article 13.1, 13.5 and 14 health claims", EFSA Journal, 9(4):2135, pp. 1-24. Doi:10.2903/j.efsa.2011.2135

EFSA (2011b): "Scientific and technical guidance for the preparation and presentation of an application for authorisation of a health claim (revision 1)", EFSA Journal, 9(5):2170, pp. 1-36. Doi:10.2903/j.efsa.2011.2170

EFSA (2016): "General scientific guidance for stakeholders on health claim applications", EFSA Journal, 14(1):4367, pp. 1-38. Doi: $10.2903 /$ j.efsa.2016.4367

EFSA (2017): "Scientific and technical guidance for the preparation and presentation of a health claim application (revision 2)", EFSA Journal, 15(1):4680, pp. 1-31. Doi:10.2903/j.efsa.2017.4680

EFSA (2018): "Scientific opinion on the xanthohumol in XERME®, a xanthohumol-enriched roasted malt extract, and protection of DNA from oxidative damage: evaluation of a health claim pursuant to Article 13(5) of Regulation (EC) No 1924/2006", EFSA Journal, 16(3):5192, pp. 1-11. Doi:10.2903/j.efsa.2018.5192

EFSA (2020): "Scientific opinion on the MenaQ7 $®$ and maintenance of the elastic properties of the arteries: evaluation of a health claim pursuant to Article 13(5) of Regulation (EC) No 1924/2006", EFSA Journal, 18(1):5949, pp. 1-10. Doi:10.2903/j. efsa.2020.5949

European Commission (2008): “Commission Regulation (EC) No 353/2008 of 18 April 2008 establishing implementing rules for applications for authorisation of health claims as provided for in Article 15 of Regulation (EC) No 1924/2006 of the European Parliament and of the Council", Official Journal of the European Union, pp. L109/11-L109/16.

European Parliament and Council (2002): "Directive 2002/46/EC of the European Parliament and of the Council of 10 June 2002 on the approximation of the laws of the Member States relating to food supplements", Official Journal of the European Communities, pp. L183/51-L183/57.

European Parliament and Council (2006): "Regulation (EC) No 1924/2006 of the European Parliament and of the Council of 20 December 2006 on nutrition and health claims made on foods", Official Journal of the European Union, pp. L404/9-L404/25.

Gregori, D. y C. E. Gafare (2012): "Multifunctional food: medical evidence and methodological notes on substantiating health claims", International Journal of Food Sciences and Nutrition, 63(S1), pp. 29-36. Doi:10.3109/09637486.2011.653553

Heaney, R. P. (2006): "Nutrition, chronic disease, and the problem of proof", The American Journal of Clinical Nutrition, 84(3), pp. 471-472. Doi:10.1093/ajcn/84.3.471

Heaney, R. P. (2008): "Nutrients, endpoints, and the problem of proof", The Journal of Nutrition, 138(9), pp. 1591-1595. Doi:10.1093/jn/138.9.1591 
Hill, A. B. (1965): "The environment and disease: association or causation?", Proceedings of the Royal Society of Medicine, 58(5), pp. 295-300.

Khedkar, S., S. Bröring y S. Ciliberti (2017): "Exploring the Nutrition and Health Claims Regulation (EC) No. 1924/2006: what is the impact on innovation in the EU food sector?", International Journal of Food Sciences and Nutrition, 68(1), pp. 10-17. Doi:10.1080/09637486.2016.1212818

Khedkar, S., S. Ciliberti y S. Bröring (2016): "The EU health claims regulation: implications for the innovation in the EU food sector”, British Food Journal, 118(11), pp. 2647-2665. Doi:10.1108/BFJ-01-2016-0021

Lenssen, K. G. M., A. Bast y A. de Boer (2018): "Clarifying the health claim assessment procedure of EFSA will benefit functional food innovation”, Journal of functional foods, 47, pp. 386-396. Doi:10.1016/j.jff.2018.05.047

Luján, J. L. y O. Todt (2018a): "The dilemmas of science for policy: scientific evidence and the consequences of regulatory options in risk and benefit assessment", EMBO reports, 19(2), pp. 194-196. Doi:10.15252/embr.201744795

Luján, J. L. y O. Todt (2018b): "Regulatory science: between technology and society", en B. Laspra y J. A. López Cerezo, eds., Spanish philosophy of technology, pp. 59-72. [Versión Adobe Acrobat Document]. Doi:10.1007/978-3-319-71958-0

Luján, J. L. y O. Todt (2021): "Evidence based methodology: a naturalistic analysis of epistemic policies in regulatory science", European Journal for Philosophy of Science, 11(26), pp. 1-19. Doi:10.1007/s13194-020-00340-7

Martínez, S. V. y A. Siani (2017): "Health claims made on food in the EU: the edge between scientific knowledge and regulatory requirements", Trends in Food Science \& Technology, 69(B), pp. 315-323. Doi:10.1016/j.tifs.2017.01.005

Mitchell, H. L., P. J. Aggett, D. P. Richardson y J. D. Stowell (2011): "Food \& health forum meeting: evidence-based nutrition", British Journal of Nutrition, 105(2), pp. 322-328. Doi:10.1017/S0007114510003296

Richardson, D. P. (2012): "Preparing dossiers: strength of the evidence and problems of proof", Proceedings of the Nutrition Society, 71(1), pp. 127-140. Doi:10.1017/S002966511100317X

Richardson, D. P. y M. Eggersdorfer (2015): “Opportunities for product innovation using authorised European Union health claims", International Journal of Food Science \& Technology, 50(1), pp. 3-12. Doi:10.1111/ijfs.12574

Sadler, M. J. (2018): “European health claims: regulatory developments", en M. J. Sadler, ed., Foods, nutrients and food ingredients with authorised EU health claims: volume 3, pp. 1-16. [Versión Adobe Acrobat Document]. Doi:10.1016/B9780-08-100922-2.00001-2

Tarabella, A. y M. Francescato (2019): “Gluten-free foods”, en A. Tarabella, ed., Food products evolution: innovation drivers and market trends, pp. 101-116. [Versión Adobe Acrobat Document]. Doi:10.1007/978-3-319-23811-1_8

Tarabella, A., E. Varese y S. Buffagni (2019): "Functional foods", en A. Tarabella, ed., Food products evolution: innovation drivers and market trends, pp. 117-142. [Versión Adobe Acrobat Document]. Doi:10.1007/978-3-319-23811-1_9

Todt, O. y J. L. Luján (2016): “¿Bueno para la salud? Un análisis de los requisitos de sustanciación científica en la regulación europea de las declaraciones de salud”, Salud pública de México, 58(3), pp. 393-398. Doi:10.21149/spm.v58i3.7899

Todt, O. y J. L. Luján (2017a): "The role of epistemic policies in regulatory science: scientific substantiation of health claims in the European Union", Journal of Risk Research, 20(4), pp. 551-565. Doi:10.1080/13669877.2015.1100661

Todt, O. y J. L. Luján (2017b): "Health claims and methodological controversy in nutrition science", Risk Analysis, 37(5), pp. 958-968. Doi:10.1111/risa.12665

Todt, O. y J. L. Luján (2021): “Constructing consumers: regulatory and methodological consequences of defining consumer preferences in European health claim regulation", Journal of Risk Research, pp. 1-12. Doi:10.1080/13669877.2021.1873825

Verhagen, H., E. Vos, S. Francl, M. Heinonen y H. van Loveren (2010): "Status of nutrition and health claims in Europe", Archives of Biochemistry and Biophysics, 501(1), pp. 6-15. Doi:10.1016/j.abb.2010.04.012

Verkerk, R. H. J. (2013): "Implementing an EU health claim converting scientific language to consumer language", Agro Food Industry Hi Tech, 24(2), pp. 32-35.

Visioli, F. (2012): “Can experimental pharmacology be always applied to human nutrition?”, International Journal of Food Sciences and Nutrition, 63(S1), pp. 10-13. Doi:10.3109/09637486.2012.665439 
